\title{
Will circumcision provide even more protection from HIV to women and men? New estimates of the population impact of circumcision interventions
}

\author{
Timothy B Hallett, ${ }^{1}$ Ramzi A Alsallaq, ${ }^{2}$ Jared M Baeten, ${ }^{3}$ Helen Weiss, ${ }^{4}$ \\ Connie Celum, ${ }^{3}$ Ron Gray, ${ }^{5}$ Laith Abu-Raddad ${ }^{2,6,7}$
}

\begin{abstract}
- Additional tables and figures are published online only. To view these files please visit the journal online (http://sti.bmj. com)
\end{abstract}

'Department of Infectious Disease Epidemiology, Imperial College London, London, UK ${ }^{2}$ Statistical Center for HIV/AIDS Research \& Prevention, Fred Hutchinson Cancer Research Center, Seattle, Washington, USA

${ }^{3}$ Departments of Global Health and Medicine, University of Washington, Seattle,

Washington, USA

${ }^{4}$ London School of Hygiene and Tropical Medicine, London, UK ${ }^{5}$ Department of Population, Family and Reproductive Health, Johns Hopkins University, Bloomberg School of Public Health, Baltimore, Maryland, USA

${ }^{6}$ Weill Cornell Medical College Oatar, Oatar Foundation -

Education City, Doha, Oatar ${ }^{7}$ Department of Public Health, Weill Cornell Medical College, Cornell University, New York, New York, USA

\section{Correspondence to} Dr Timothy B Hallett, Imperial College London, St Mary's Campus, Norfolk Place, London W2 1PG, UK:

timothy.hallett@imperial.ac.uk

Accepted 24 August 2010 Published Online First

21 October 2010

\section{ABSTRACT}

Background Mathematical modelling has indicated that expansion of male circumcision services in high HIV prevalence settings can substantially reduce populationlevel HIV transmission. However, these projections need revision to incorporate new data on the effect of male circumcision on the risk of acquiring and transmitting HIV.

Methods Recent data on the effect of male circumcision during wound healing and the risk of HIV transmission to women were synthesised based on four trials of circumcision among adults and new observational data of HIV transmission rates in stable partnerships from men circumcised at younger ages. New estimates were generated for the impact of circumcision interventions in two mathematical models, representing the HIV epidemics in Zimbabwe and Kisumu, Kenya. The models did not capture the interaction between circumcision, HIV and other sexually transmitted infections.

Results An increase in the risk of HIV acquisition and transmission during wound healing is unlikely to have a major impact of circumcision interventions. However, it was estimated that circumcision confers a $46 \%$ reduction in the rate of male-to-female HIV transmission. If this reduction begins 2 years after the procedure, the impact of circumcision is substantially enhanced and accelerated compared with previous projections with no such effect-increasing by $40 \%$ the infections averted by the intervention overall and doubling the number of infections averted among women.

Conclusions Communities, and especially women, may benefit much more from circumcision interventions than had previously been predicted, and these results provide an even greater imperative to increase scale-up of safe male circumcision services.

\section{BACKGROUND}

Since three randomised controlled trials demonstrated that adult male circumcision reduces men's chance of HIV acquisition by about $60 \%,{ }^{1-3}$ there has been substantial interest in developing and expanding services that provide male circumcision as an additional HIV prevention strategy. ${ }^{4}$ An important part of the planning and strategic decision-making process for expanding circumcision services has been quantifying the expected impact such interventions might have on the rate of new HIV infections in populations, and several mathematical models have been developed to generate estimates. $^{5-9}$ To guide HIV prevention policy decisions, these estimates are compared with others that evaluate different prevention strategies, such as scaling-up testing and counselling services, ${ }^{10}{ }^{11}$ campaigns with messages about abstinence, condom use and reducing multiple partners, ${ }^{12}$ 13 and universal HIV testing with immediate initiation of antiretroviral therapy (ie, 'Universal Test and Treat'). ${ }^{14} 15$

Previous modelling of the impact of male circumcision on population-level HIV had two important limitations: lack of data about the rate of HIV transmission (both female-to-male and maleto-female) before the wound has healed, and the long-term effect of male circumcision on risk of male-to-female transmission. We hypothesised that these limitations resulted in substantial imprecision in the estimate of the impact of circumcision interventions on HIV spread, both overall and especially among women.

Recently, new data have become available to address these limitations. A pooled analysis from the three trials evaluating the effect of male circumcision on female-to-male HIV transmission found that sex before the wound has healed was associated with a transient increased risk of HIV acquisition in men. ${ }^{16}$ In addition, two recent studies have reported on the relationship between male circumcision and maleto-female HIV transmission. The first, a randomised trial of circumcision from Uganda, found no shortterm benefit of circumcision (ie, to 24 months after the operation) and some suggestion of increased HIV transmission risk to women from circumcised HIVinfected men who resumed sex before the wound was healed. ${ }^{17}$ The second, an observational analysis from a cohort of 3408 HIV-discordant couples from 14 sites in eastern and southern Africa followed for up to 2 years, found that male-to-female HIV transmission risk was $41 \%$ lower from circumcised men compared with uncircumcised men (relative transmission rate of 0.59 ( $95 \%$ CI 0.31 to 1.13 ); the corresponding estimate adjusted for viral load is: 0.60 (95\% CI 0.31 to 1.16$)$ ). ${ }^{18}$ These data mirror those from an earlier observational study in Rakai, Uganda that found a relative male-to-female HIV transmission rate of 0.41 (95\% CI 0.10 to 1.14) in couples with circumcised versus uncircumcised HIVinfected men. ${ }^{19}$ Together, these data suggest that association between circumcision and male-tofemale HIV transmission risk is influenced by the timing of circumcision, with recent circumcision offering no benefit, but circumcision at an earlier age (and thus a longer interval between surgery to sex) significantly reducing HIV transmission risk to female partners. 
To explore the implications of these new observations for the estimates of the potential impact of circumcision interventions, we adapted two previous published mathematical models-by Hallett et $a l^{6}$ for Zimbabwe and Alsallaq et al ${ }^{20}$ for Kisumu, Kenya.

\section{METHODS}

Full descriptions of the two mathematical models have already been published, ${ }^{620}$ and brief descriptions are provided here.

\section{Model 1: Zimbabwe (Hallett et al)}

This model is a deterministic compartmental representation of the heterosexual spread of HIV in Zimbabwe, with HIV prevalence of $\sim 20 \%$ and initially low rates of circumcision. ${ }^{21}$ To capture the heterogeneity in the number of sexual partners, men and women in the model were stratified into risk groups. Men and women form partnerships so that it is more likely that high-risk individuals form partnerships with one another. Published data from eastern Zimbabwe ${ }^{22}$ were used to inform these parameters specifying sexual behaviour (although the broad behavioural patterns are similar to reports in other settings). Based on observational data from other longitudinal studies, ${ }^{23}$ the course of infection is represented by individuals progressing through several stages: acute infection (short duration, high infectiousness), latent infection (long duration, low infectiousness) and pre-AIDS (short duration, high infectiousness). The Zimbabwe model was fitted to both the antenatal clinic prevalence data and the Demographic and Health Surveys prevalence estimate.

\section{Model 2: Kisumu, Kenya (Alsallaq et al)}

This model is a deterministic compartmental representation of the heterosexual spread of HIV in Kisumu, Kenya calibrated by three sets of prevalence data: the Four City study population survey data, ${ }^{24}$ the Kenya AIDS indicator survey data for Kisumu, ${ }^{25}$ and the ANC surveillance data. ${ }^{26}{ }^{27}$ Before the intervention, HIV prevalence is $\sim 15 \%$, and $25 \%$ of adult men are circumcised. ${ }^{28} \mathrm{~A}$ hierarchy of effective rates of partnership formation were used as a surrogate of sexual risk to stratify the population into four sexual-risk groups. The effective rates of partnership formation for females were assumed to balance those for males, which were adjusted by fitting the model to empirical behaviour data of Kisumu. ${ }^{24-27}$ Other parameters were informed by empirical data, including transmission probabilities per coital act per HIV stage, ${ }^{23} 29$ frequency of coital acts per HIV stage, ${ }^{23}$ duration of sexual partnerships, ${ }^{30}$ duration of each HIV stage, ${ }^{23} 3132$ and fraction of the population initially in each risk group ( $\mathrm{R} \mathrm{H}$ Gray, personal communication, 2008)..$^{30} 33$
An important limitation is that neither model explicitly accounts for possible interactions between circumcision and other sexually transmitted infections (STIs).

\section{Parameterisation of circumcision and intervention}

In both models, seven independent parameters specify the effect of circumcision on transmission (table 1; ranges for sensitivity analysis are presented in the online supplementary table S1). The reduction in male-to-female transmission of HIV was assumed to begin 2 years after the operation in order to be consistent with the observational data, showing a large effect, ${ }^{18} 19$ and trial data, which did not find evidence for such an effect within 2 years of the operation (recognising that the trial was not sufficiently powered to do $\mathrm{so}^{17}$ ). In the modelled interventions, unless otherwise specified, a constant rate of operations was assumed such that $50 \%$ of non-circumcised men in a given population were circumcised in the first 10 years after the start of the intervention (HIV negative and positive men had the same chance of being circumcised).

\section{RESULTS}

Firstly, we explored the potential impact of the risk of HIV acquisition and transmission being higher during the wound healing period. In a hypothetical intervention that reduced the proportion of the population that are uncircumcised to $50 \%$ over 10 years and in which no men resume sex during wound healing, the HIV incidence rate (new infections per person-years at risk) in the whole population after 10 years is reduced by $20.5 \%$ in Zimbabwe (0\% circumcised before intervention) and by $7.4 \%$ in Kisumu (25\% circumcised before intervention). Alternatively, making the most pessimistic assumptions that all men remain sexually active throughout the wound healing period and the mean duration of wound healing is as long as six weeks, the incidence rate is still projected to decrease by $19 \%$ in Zimbabwe and $6.2 \%$ in Kisumu over the same period. Differences between the simulations decrease over time, so the long-term effect of the elevated chance of HIV acquisition and transmission during the circumcision wound healing period is likely to be even less.

Next we conducted a meta-analysis of data from the two independent observational cohorts ${ }^{19}{ }^{36}$ on the long-term effect of male circumcision on male-to-female HIV transmission. The overall fixed-effect point-estimate hazard rate was 0.54 (95\% CI 0.31 to 0.96$)(p=0.04)$, indicating $46 \%$ reduction in transmission rate from 2 years after the operation. There was no evidence for heterogeneity in effect size $(p=0.61)$.

On the basis of the meta-analysis results, we explored the impact of the reduction in the rate of transmission from

Table 1 Parameters specifying the effect of male circumcision on the rate of HIV transmission (ranges used in the uncertainty analysis are presented in table $S 1$ in the online technical appendix)

\begin{tabular}{|c|c|c|}
\hline Parameter description & Value & Source \\
\hline Mean duration of wound healing period & 14 days (95\% healed within 6 weeks). & 34 \\
\hline $\begin{array}{l}\text { Change in the rate of female-to-male HIV transmission before wound is healed } \\
\text { versus before circumcision }\end{array}$ & Increases 3-fold & 16 \\
\hline $\begin{array}{l}\text { Change in rate of HIV male-to-female HIV transmission before wound is healed } \\
\text { versus before circumcision }\end{array}$ & Increases 3.5-fold & 17 \\
\hline Proportion of men who remain sexually active during the wound healing period & $13 \%$ & 16 \\
\hline $\begin{array}{l}\text { Change in rate of female-to-male HIV transmission after the wound has healed } \\
\text { versus before circumcision }\end{array}$ & Decreases by $65 \%$ & 35 \\
\hline $\begin{array}{l}\text { Change in rate of male-to-female HIV transmission before } 2 \text { years after the operation } \\
\text { versus before circumcision }\end{array}$ & No effect & 17 \\
\hline $\begin{array}{l}\text { Change in rate of male-to-female HIV transmission from } 2 \text { years after the operation } \\
\text { versus before circumcision }\end{array}$ & $\begin{array}{l}\text { We estimated this after performing a meta-analysis of data from the } \\
\text { two studies among HIV sero-discordant couples that measured this effect }\end{array}$ & 1819 \\
\hline
\end{tabular}


Figure 1 Estimated reduction in incidence following circumcision interventions among the whole adult population $(A, B)$ and women $(C, D)$, in Zimbabwe (model 1$)$ and Kisumu, Kenya (model 2), respectively. It is assumed that circumcision reduces the chance of male-to-female transmission by $0 \%$ (solid line) and $46 \%$ (dashed lines). For the other assumptions, see the text.
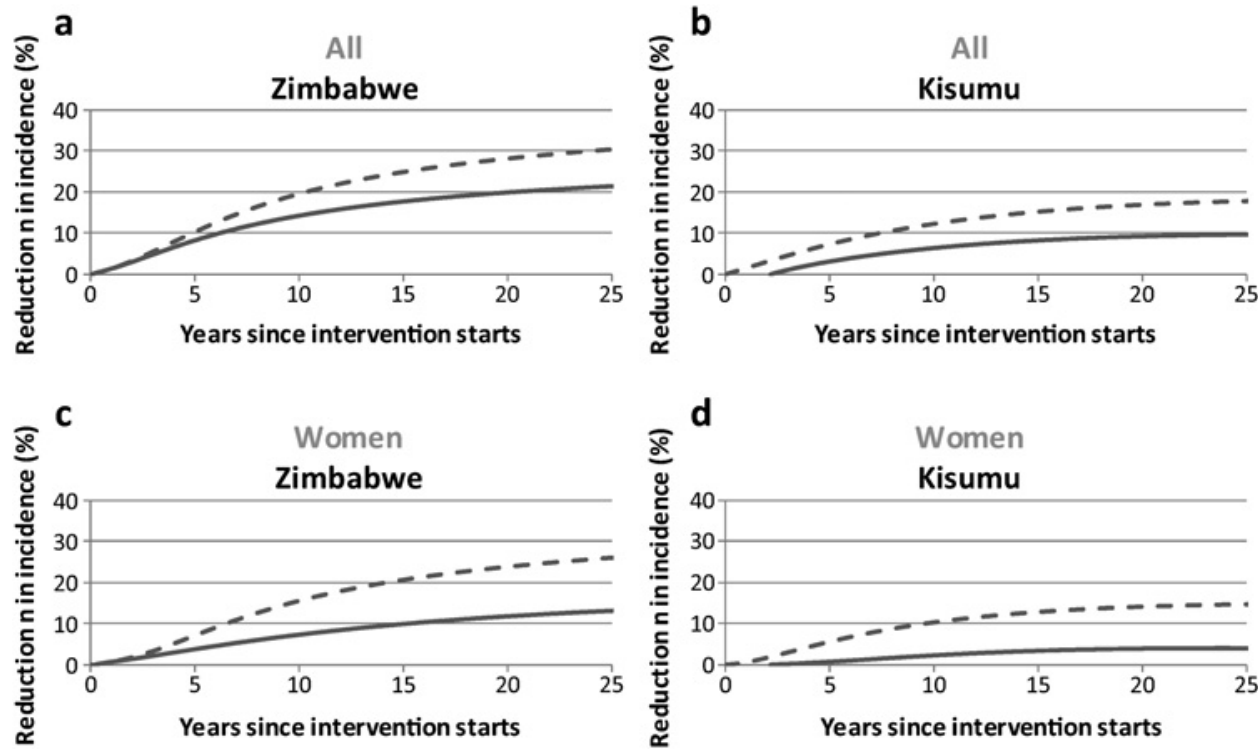

circumcised HIV-infected men to their uninfected female partners, after the wound healing period and the subsequent 2 years (as defined in the Methods). Assuming first no effect of circumcision on male-to-female transmission and using best estimates for the duration of wound healing (table S1), our simulations suggest that the incidence rate would decrease by $19.8 \%$ in Zimbabwe or $9.4 \%$ in Kisumu after 20 years. Then, assuming an estimated $46 \%$ reduction in male-to-female transmission rate associated with circumcision, starting 2 years after the procedure, corresponding estimates for the reduction in incidence are $28.0 \%$ (Zimbabwe) and 16.8\% (Kisumu) (figure 1A,B). This is an increase in epidemiological impact of at least $40 \%$ overall.

As expected, the reduction in incidence with the male-tofemale transmission effect is most enhanced among women (figure 1C,D). Without an effect of circumcision on male-tofemale HIV transmission, incidence is reduced among women by $11.7 \%$ in Zimbabwe or $3.9 \%$ in Kisumu after 20 years; but, with the direct effect on male-to-female transmission, incidence is instead reduced by $23.7 \%$ (Zimbabwe) or $13.9 \%$ (Kisumu). Thus the epidemiological impact among women at least almost doubles.

The number of operations required for each HIV infection averted is also substantially reduced if there is a long-term reduction in male-to-female transmission of HIV as a result of male circumcision: in Zimbabwe, from 11 operations per infection averted after 20 years if there is no effect on transmission to eight operations (down 28\%); and, in Kisumu, from 14 operations per infection to eight operations over the same period (down 41\%).

The multivariate uncertainty was used to determine how the uncertainty in the estimated effects of circumcision propagates to the projections in epidemiological impact (Zimbabwe model only, but qualitatively similar results would be expected for the Kisumu model). With the original assumptions (no effect of wound healing and no effect on male-to-female HIV transmission), the uncertainty intervals for the reduction in incidence after 20 years are $15-32 \%$ overall and $9-21 \%$ among women. The new empirical data (table S1) support corresponding updated estimates of $22-43 \%$ reduction in incidence overall, and a $15-39 \%$ reduction among women (figure 2 ).

If there is no effect of circumcision on male-to-female transmission and a moderate degree of increased risk behaviour following the operation, then it is possible that HIV incidence among women and the population overall could increase following the intervention (online supplementary figure S1)a serious adverse outcome. However, assuming a $46 \%$ reduction in the transmission rate with circumcision (2 years after the operation), then our modelling suggests that even very high degrees of increased risk among circumcised men would not lead to increases in incidence overall under the intervention.

\section{DISCUSSION}

Our synthesis of new data about the effect of circumcision on female-to-male and male-to-female HIV transmission provides an even greater imperative to increase scale-up of circumcision interventions to prevent HIV. ${ }^{37} 38$ We found that (a) a transient increased risk of HIV transmission (in both directions) during
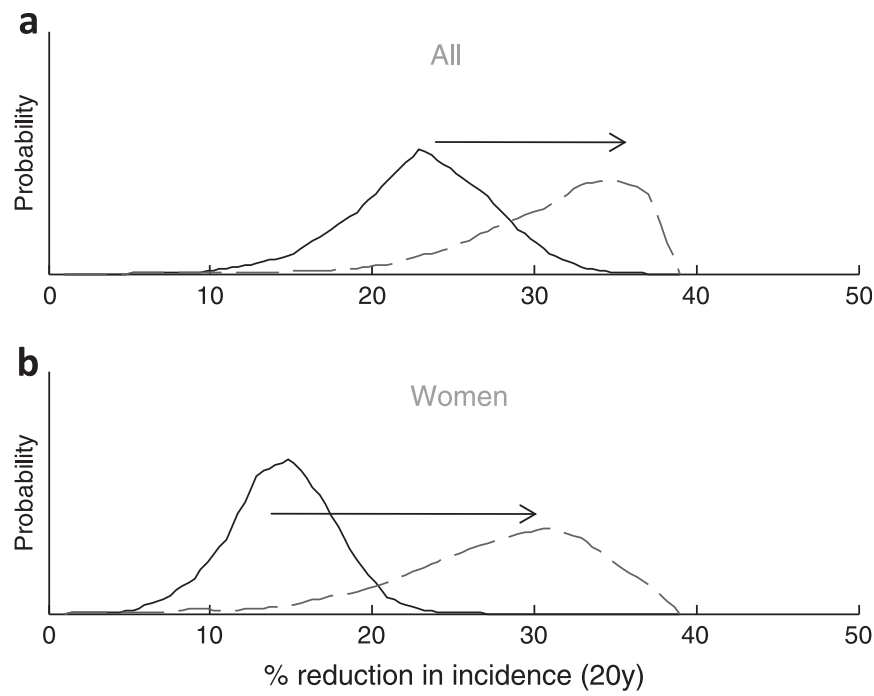

Figure 2 Multivariate uncertainty analysis of the reduction in HIV incidence 20 years after a circumcision intervention starts, among the whole adult population (A) and women (B). The solid line shows the estimate making the original assumptions (ie, only the effect of circumcision on the chance of acquisition after the wound heals); the dashed line shows the updated estimates using the new information (ie, includes effect of circumcision on acquisition/transmission during wound healing and effect on male-to-female transmission after wound healing). Distributions for the parameters are shown in the online supplementary table S1. 
the circumcision wound healing period resulted in only a negligible effect on HIV incidence rate in populations, $(b)$ potential long-term reduced risk of HIV transmission from HIV-infected circumcised men amplified the benefits of circumcision implementation, and (c) the benefits of circumcision in these scenarios were particularly great for women.

While short-term increased risk for both female-to-male and male-to-female HIV transmission may occur while the wound heals, this effect is very unlikely to materially affect the impact of circumcision interventions at the population level. Nonetheless, careful communication strategies should still be used to promote abstinence for at least 2 months after the operation to minimise the chance of HIV transmission to female partners.

Our meta-analysis of the best available cohort data suggests that male circumcision reduces the chance of male-to-female HIV transmission with a latent interval of at least 2 years, ${ }^{18} 19$ and that this substantially amplifies the potential impact of circumcision interventions in populations. Our new projections for the reduction in HIV incidence following circumcision interventions are $40 \%$ greater in Zimbabwe and $79 \%$ greater for Kisumu, and the number of operations required per infection averted is reduced by more than $25 \%$, when the overall benefit in reduced HIV male-to-female transmission was included. This increases the already attractive cost-saving potential of circumcision interventions. ${ }^{83}$ (In the online supplementary text S1, we have also provided a simple formula for making a calculation of operations per infection averted-ie, generalised to other settings.) Indeed, the combination of the two separate effects of circumcision (reducing the chance that men acquire HIV infection and reducing the chance that infected men pass on HIV infection) leads to a more powerful effect than would be expected on the basis of considering the two effects separately (figure S2).

Importantly, our simulations show very significantly that the benefit of circumcision interventions to women is greatly increased. Although it was previously expected that women would benefit modestly from male circumcision implementation (as fewer of their sexual partners would be infected ${ }^{6}$ ), these new projections show that women could also receive a direct benefit from circumcision. Earlier work had predicted that the population impact of circumcision intervention would be sensitive to this factor. ${ }^{39} 40$ If our estimate about the long-term effect of circumcision on male-to-female transmission is correct, then women in stable partnerships with infected men would receive a degree of protection from circumcision similar to that received by men in partnerships with infected women. We note, however, that the standard of evidence for an effect on male-tofemale transmission (meta-analysis of two cohort studies) is much less than that for the effect of circumcision on men's chance of acquiring HIV infection (three randomised trials and more than 36 observational studies ${ }^{35}$ ). Our results do not indicate that HIV-infected men should be targeted for circumcision, as most of the impact of the reduction in male-to-female transmission comes through reducing onward transmission of 'break-through' infections among men circumcised when they were not infected (if only uninfected men are circumcised in the intervention, the eventual reduction in incidence is increased by $30 \%$ overall and $66 \%$ for women, compared with $44 \%$ and $95 \%$, respectively, if HIV-infected men are circumcised too). Thus, our findings support the current UNAIDS recommendations that men seeking circumcision who are already HIV infected be discouraged but not refused the operation.

In addition, earlier concerns about women being exposed to increased risk due to risk compensation among circumcised men are substantially allayed. Our calculations show that it is unlikely that risk compensation following circumcision will undermine the benefits for either men or women.

We adapted two previously developed mathematical models, both of which make a number of simplifying assumptions about the pattern of sex partner contact and HIV natural history, but which have nevertheless proved useful in developing recommendations and strategic decision-making in male circumcision interventions. $^{20} 374041$ Application of the same analysis to two independently constructed models, parameterised for different settings, also indicates that our findings are not specific to one type of modelling approach or one population. There are similarities in the model structure (both are deterministic compartmental transmission models which include stratification by risk group and an account of mixing in sexual contact between risk groups), but substantial differences remain, besides parameterisation, such as the way that the force of infection is calculated and the assumption made about entry and exit to different risk categories. The impact of circumcision interventions is somewhat less in Kisumu (model 2) than in Zimbabwe (model 1), because the basic reproductive ratio is estimated to be higher in Kisumu (this reduces the impact of many types of intervention $^{42}$ ) and $25 \%$ of men in Kisumu are already circumcised. ${ }^{20}$ These results should not be interpreted as literal forecasts for either setting, as the rate of scale-up of circumcision that will be achieved is far from certain, and the imprecision in the specification of the epidemiological conditions was not fully quantified. ${ }^{43}$

An important limitation is that neither model explicitly accounts for possible interactions between circumcision and other STIs. ${ }^{44}$ Therefore the benefits of circumcision interventions in reducing the incidence of other STIs cannot be quantified here. Any indirect effects on HIV incidence that accrue through reduced STI prevalence will also not be captured in the models, so our results may in fact underestimate the impact of the interventions. However, earlier work indicates that the strength of those indirect effects is expected to be small relative to the direct effect of circumcision on HIV acquisition (and transmission). ${ }^{44} 45$

Indirect observational data have produced conflicting evidence on the effect of circumcision on male-to-female HIV transmission, but most studies were severely limited by a lack of knowledge on the circumcision or infection status of men. ${ }^{37} \mathrm{~A}$ recent meta-analysis included data from the Rakai randomised controlled trial among HIV-infected men ${ }^{17}$ and six longitudinal studies, with $95 \%$ CI 0.53 to 1.36 (point estimate 0.80 ). ${ }^{46}$ In this paper, we have restricted analyses to the only two longitudinal studies that directly measured rates of transmission from HIV-infected men of known circumcision status at the time of study entry to their regular partners. ${ }^{18} 19$ Both found evidence for a reduction in transmission, although small numbers limited statistical significance in each study when analysed separately. Our meta-analysis of these highly comparable datasets, however, provides statistically significant evidence of a true effect. The uncertainty in the estimated effect size was, nevertheless, fully represented in this analysis (figure 2). The Rakai trial $^{17}$ showed no efficacy in the 2 years of follow-up of female partners after their male partners were circumcised, suggesting that it may take time for keratinisation of the scar and glans or other biological changes to develop, and here we assumed that the effect developed 2 years after the operation. Encouragingly, more data will become available from on-going HIV vaccine trials ${ }^{47-49}$ and other studies, which will help to hone our knowledge about the effect of circumcision and refining model projections. 


\section{Key messages}

- Circumcision may confer a $46 \%$ reduction in the rate of HIV transmission from circumcised men to their female partners.

- Assuming a reduction in male-to-female transmission, the projected impact of circumcision on HIV spread is substantially enhanced, especially for women.

- An increase in the risk of acquisition and transmission of HIV during circumcision wound healing is unlikely to have a major effect on the population-level impact of circumcision interventions.

In conclusion, projections for the impact of circumcision interventions on population-level HIV may need to be dramatically revised: the impact of male circumcision implementation could be realised much sooner and with greater cost-efficiency than had previously been thought. It is also crucial to recognise and communicate that, although male circumcision is an intervention applied to men, it brings substantial benefits for women as well. Premature resumption of sexual activity before the wound is healed or 'compensatory' increases in risk following circumcision are both unlikely to substantively undermine the benefits of male circumcision on HIV incidence among women or men.

Acknowledgements We thank The Wellcome Trust (TBH) and the US National Institutes of Health (grant 1R01Al083034) and Oatar National Research Fund (NPRP 08-068-3-024) (TBH and LA-R) for funding support. The funders had no role in study design, data collection and analysis, decision to publish, or preparation of the manuscript.

Funding Wellcome Trust $+\mathrm{NIH}$.

Competing interests None.

Provenance and peer review Not commissioned; externally peer reviewed.

\section{REFERENCES}

1. Auvert B, Taljaard D, Lagarde E, et al. Randomized, controlled intervention trial of male circumcision for reduction of HIV infection risk: the ANRS 1265 Trial. PLoS Med 2005;2:e298.

2. Gray RH, Kigozi G, Serwadda D, et al. Male circumcision for HIV prevention in men in Rakai, Uganda: a randomised trial. Lancet 2007;369:657-66

3. Bailey RC, Moses S, Parker CB, et al. Male circumcision for HIV prevention in young men in Kisumu, Kenya: a randomised controlled trial. Lancet 2007:369:643-56.

4. WHO, UNAIDS. New Data on Male Circumcision and HIV Prevention: Policy and Programme Implications. http://www.data.unaids.org/pub/Report/2007/ mc recommendations en.pdf (accessed 3 Feb 2011).

5. Williams BG, Lloyd-Smith JO, Gouws E, et al. The potential impact of male circumcision on HIV in Sub-Saharan Africa. PLoS Med 2006;3:e262.

6. Hallett TB, Singh K, Smith JA, et al. Understanding the impact of male circumcision interventions on the spread of HIV in southern Africa. PLOS ONE 2008;3:e2212.

7. White RG, Glynn JR, Orroth KK, et al. Male circumcision for HIV prevention in sub-Saharan Africa: who, what and when? AIDS 2008:22:1841-50.

8. Kahn JG, Marseille E, Auvert B. Cost-effectiveness of male circumcision for HIV prevention in a South African setting. PLoS Med 2006;3:e517.

9. UNAIDS/WHO/SACEMA Expert Group on Modelling the Impact Cost of Male Circumcision for HIV Prevention. Male circumcision for hiv prevention in high hiv prevalence settings: what can mathematical modelling contribute to informed decision making? PLoS Med 2009;6:e1000109.

10. WHO, UNAIDS. Guidance on provider-initiated HIV testing and counselling in health facilities. http://www.who.int/hiv/topics/vct/PITCguidelines.pdf laccessed 3 Feb 2011).

11. Hallett TB, Dube S, Cremin I, et al. The role of testing and counselling for hiv prevention and care in the era of scaling-up antiretroviral therapy. Epidemics 2009;1:77-82

12. Halperin DT, Steiner MJ, Cassell MM, et al. The time has come for common ground on preventing sexual transmission of HIV. Lancet 2004;364:1913-15.

13. Shelton JD. Why multiple sexual partners? Lancet 2009;374:367-9.

14. Granich RM, Gilks CF, Dye C, et al. Universal voluntary HIV testing with immediate antiretroviral therapy as a strategy for elimination of HIV transmission: a mathematical model. Lancet 2008:373:48-57.
15. Dodd PJ, Garnett GP. Hallett TB. Examining the promise of HIV elimination by 'test and treat' in hyperendemic settings. AIDS 2010;24:729-35

16. Mehta SD, Gray RH, Auvert B, et al. Does sex in the early period after circumcision increase HIV-seroconversion risk? Pooled analysis of adult male circumcision clinical trials. AIDS 2009;23:1557-64.

17. Wawer MJ, Makumbi F, Kigozi G, et al. Circumcision in HIV-infected men and its effect on HIV transmission to female partners in Rakai, Uganda: a randomised controlled trial. Lancet 2009;374:229-37.

18. Baeten JM, Donnell D, Kapiga SH, et al. Male circumcision and risk of male-tofemale HIV-1 transmission: a multinational prospective study in African HIV-1serodiscordant couples. AIDS 2009;24:737-44.

19. Gray RH, Kiwanuka N, Quinn TC, et al. Male circumcision and HIV acquisition and transmission: cohort studies in Rakai, Uganda. Rakai Project Team. AIDS 2000:14:2371-81.

20. Alsallaq R, Cash $B$, Weiss $H$, et al. Quantitative assessment of the role of male circumcision in HIV epidemiology at the population level. Epidemics 2009;1:139-52

21. Hallett TB, Gregson S, Gonese E, et al. Assessing evidence for behaviour change affecting the course of HIV epidemics: a new mathematical modelling approach and application to data. Zimbabwe Epidemics 2009;1:108-17.

22. Gregson S, Nyamukapa CA, Garnett GP, et al. Sexual mixing patterns and sex-differentials in teenage exposure to HIV infection in rural Zimbabwe. Lancet 2002;359:1896-903.

23. Wawer MJ, Gray RH, Sewankambo NK, et al. Rates of HIV-1 transmission per coital act, by stage of HIV-1 infection, in Rakai, Uganda. J Infect Dis 2005:191:1403-9.

24. Buvé A, Carael M, Hayes RJ, et al. The multicentre study on factors determining the differential spread of HIV in four African cities: summary and conclusions. AIDS 2001;15(Suppl 4):S127-31.

25. National AIDS and STI Control Programme MoH. Kenya AIDS Indicator Survey 2007: Preliminary Report. Nairobi, Kenya: National AIDS and STI Control Programme $\mathrm{MoH}, 2008$.

26. UNAIDS/WHO. AIDS epidemic update 2004. 2004

27. WHO/AFRO. HIVIAIDS Epidemiological Surveillance Update for the WHO African Region 2002 Country Profiles. 2002

28. Auvert B, Buvé A, Lagarde E, et al. Male circumcision and HIV infection in four cities in sub-Saharan Africa. AIDS 2001;15(Suppl 4):S31-40.

29. Abu-Raddad LJ, Patnaik P, Kublin JG. Dual infection with HIV and malaria fuels the spread of both diseases in sub-Saharan Africa. Science 2006;314:1603-6.

30. Morison L, Weiss HA, Buve A, et al. Commercial sex and the spread of HIV in fou cities in sub-Saharan Africa. AIDS 2001;15(Suppl 4):S61-9.

31. Morgan D, Whitworth J. The natural history of HIV-1 infection in Africa. Nat Med 2001:7:143-5.

32. UNAIDS. UNAIDS Reference Group on Estimates, Modelling and Projections. UNAIDS, 2007

33. Ferry B, Carael M, Buve A, et al. Comparison of key parameters of sexual behaviou in four African urban populations with different levels of HIV infection. AIDS 2001:15(Suppl 4):S41-50

34. Kigozi G, Gray RH, Wawer MJ, et al. The safety of adult male circumcision in HIV-infected and uninfected men in Rakai, Uganda. PLoS Med 2008;5:e116.

35. Weiss HA, Halperin D, Bailey RC, et al. Male circumcision for HIV prevention: from evidence to action? AIDS 2008;22:567-74.

36. Baeten J, Donnell D, Inambao M, et al. Male Circumcision and Risk of Male-toFemale HIV-1 Transmission: A Multinational Prospectiv Study. 5th International AIDS Society Conference on HIV Pathogenesis, Treatment and Prevention. Cape Town, South Africa, 19-22 July, 2009.

37. Kim S. Evaluation of impact of adult male circumcision programs on HIV incidence and prevalence: Current research, gaps in knowledge and recommendations for additional research. Report of a roundtable discussion convened by the forum for collaborative HIV research in collaboration with the Bill \& Melinda gates foundation World Health Organization, and UNAIDS. Washington, DC. Forum for Collaborative HIV Research, 2008. http://www.hivforum.org/storage/hivforum/documents/MC2/ mc2\%20report\%20final.pdf (accessed 3 Feb 2011).

38. Hankins C. Male circumcision for HIV prevention: Operational research priorities. London: International Society for Sexually Transmitted Diseases Research, 2009.

39. Gray RH, Li X, Kigozi G, et al. The impact of male circumcision on HIV incidence and cost per infection prevented: a stochastic simulation model from Rakai, Uganda. AIDS 2007;21:845-50.

40. Manicaland HIV/STD Prevention Project. Mathematical modelling tools in the response to the HIV epidemic in Zimbabwe. London \& Harare, Zimbabwe: Imperial College London \& Biomedical Research and Training Institute, 2008.

41. UNAIDS/WHO/SACEMA Expert Group on Modelling the Impact Cost of Male Circumcision for HIV Prevention. Male circumcision for HIV prevention in high HIV prevalence settings: what can mathematical modelling contribute to informed decision making? PLoS Med 2009;6:e1000109.

42. Garnett GP, Anderson RM. Strategies for limiting the spread of HIV in developing countries: conclusions based on studies of the transmission dynamics of the virus. J Acquir Immune Defic Syndr Hum Retrovirol 1995:9:500-13.

43. Garnett GP, Cousens S, Hallett TB, et al. Mathematical models in the evaluation of health programmes. Lancet. In press.

44. Desai K, Boily MC, Garnett GP, et al. The role of sexually transmitted infections in male circumcision effectiveness against HIV-insights from clinical trial simulation. Emerg Themes Epidemiol 2006;3:19. 
45. Boily MC, Desai K, Masse B, et al. Incremental role of male circumcision on a generalised HIV epidemic through its protective effect against other sexually transmitted infections: from efficacy to effectiveness to population-level impact. Sex Transm Infect 2008;84(Suppl 2):ii28-34.

46. Weiss HA, Hankins CA, Dickson K. Male circumcision and risk of HIV infection in women: a systematic review and meta-analysis. Lancet Infect Dis 2009;9:669-77.

47. Gray GE, Kublin JG. Safety and Efficacy of a Three-Dose Regimen of an Adenoviral HIV Vaccine (MRKAd5 HIV-1 Gag/Pol/Nef) in HIV Uninfected South African Adults. 2007. http://www clinicaltrials.gov/ct/show/NCT00413725; isessionid=A6758E80AEA5A1111E 76F301C01DE11E?order=2 (accessed 3 Feb 2011).

48. Pitisuttithum $\mathbf{P}$, Gilbert $\mathrm{P}$, Gurwith $\mathrm{M}$, et al. Randomized, double-blind, placebocontrolled efficacy trial of a bivalent recombinant glycoprotein $120 \mathrm{HIV}-1$ vaccine among injection drug users in Bangkok, Thailand. J Infect Dis 2006;194:1661-71.

49. Rerks-Ngarm S, Brown AE, Khamboonruang C, et al. HIV/AIDS preventive vaccine 'prime-boost' phase III trial: foundations and initial lessons learned from Thailand. AIDS 2006;20:1471-9.

\section{Specialty induction for junior doctors in genitourinary/HIV medicine using an e-learning tool}

Junior doctors' specialty induction within our genitourinary medicine (GUM)/HIV department has traditionally comprised of a lecturebased programme. This covers local guidelines, runs over 2 days, three times a year, requires significant coordination and input from the multidisciplinary team and occupies our main lecture theatre, thereby interrupting the department's educational agenda.

Training Tracker is an e-learning tool used by over 80 NHS trusts, for staff trust induction and statutory/mandatory training. Chelsea and Westminster Hospital is one such trust. Using this software, we devised, piloted and evaluated a GUM/HIV specialty online induction programme for junior doctors.

In August 2009, 12 doctors joining the specialty received the traditional lecturebased induction and completed a new e-induction programme using Training Tracker. Doctors then returned a feedback questionnaire anonymously.

The e-programme consisted of over 30 modules that delivered similar content to the traditional lectures. The modules were completed during weekly educational and non-clinical sessions over the first 4-6 weeks.
The website was accessed using a unique username and password, from work or remotely if preferred. Assistance with modules, if required, was provided by the participant's educational supervisor.

Twelve junior doctors (four specialist registrars, two foundation year trainees, two GP trainees, two core medical trainees and two trust grade doctors) piloted the e-learning tool. All completed the evaluation form.

Doctors rated their overall level of learning from Training Tracker at 7.5/10 (median) $(0=$ nil, $10=$ excellent, range $6-9)$. Doctors agreed that they could log on $(100 \%)$, navigate the site without difficulty $(100 \%)$ and that the content was useful $(11 / 12,92 \%)$, relevant $(100 \%)$ and of appropriate depth $(11 / 12,92 \%)$ and pitch $(10 / 11,91 \%)$ for their needs.

Only one doctor found the lecture induction preferable to the e-programme. Ten doctors $(83 \%)$ found the e-modules covered everything sufficiently well.

E-learning is rapidly growing as a form of delivering training. Learning times can be reduced by $40-60 \%$ and application to the job is enhanced by $25 \%$ compared with traditional teaching methods. ${ }^{12}$

Using e-learning for GUM/HIV specialty induction was found to be acceptable, user friendly and could partly/wholly replace a lecture-based programme. Its use released valuable staff time and space and catered for a large intake of doctors. Added benefits include: on-demand availability, caters for staff joining outside the classic intake times, reduced information overload, enables participants to self-pace and thus caters for a range of learners, built-in anti-cheating deterrents, consistent delivery of content and universal interactivity.

\section{S Day, M Rayment, M Mohabeer}

Directorate of HIV/GUM, Chelsea and Westminster Hospital, London, UK

Correspondence to Dr Sara Day, Directorate of HIV/ GUM, Chelsea and Westminster Hospital, 4th Floor St Stephens Centre, 369 Fulham Road, London, SE10 9NH, UK; saralouiseday@googlemail.com

Competing interests None declared.

Patient consent Obtained.

Provenance and peer review Not commissioned; not externally peer reviewed.

Accepted 6 December 2010

Published Online First 10 January 2011

Sex Transm Infect 2011;87:93.

doi:10.1136/sti.2010.048124

\section{REFERENCES}

1. Hall B. Web-based Training Cookbook. New York: Wiley Computing Publishing, 1997:108.

2. Fletcher JD. Effectiveness and cost of interactive videodisc instruction in defense training and education. Multimed Rev 1991;2:33-42. 